\title{
Some Results Related Integral Inequalities on Time Scales
}

\author{
B. I. Yaşar and A. Tuna \\ Department of Mathematics, Faculty of Science and Arts \\ University of Gazi, Beşevler, 06500, Ankara, Turkey \\ irfany@gazi.edu.tr \\ adnantuna@gazi.edu.tr
}

\begin{abstract}
In this article we study some results related to integral inequalities by using Hölder's inequality on time scales.

Mathematics Subject Classification: Primary 26D15, 39A10

Keywords: Integral inequalities; Hölder's inequality; Cauchy-Schwarz inequality; Time scales
\end{abstract}

\section{Introduction}

The theory of dynamic equations on time scales(aka measure chains) was introduced by Hilger [3] with the motivation of providing a unified approach to continuous and discrete analysis. The generalized derivative or Hilger derivative $f^{\triangle}(t)$ of a function $f: \mathbb{T} \rightarrow \mathbb{R}$, where $\mathbb{T}$ is a so-called "time scale" (an arbitrary closed nonempty subset of $\mathbb{R}$ ) becomes the usual derivative when $\mathbb{T}=\mathbb{R}$, that is $f^{\triangle}(t)=f^{\prime}(t)$. On the other hand, if $\mathbb{T}=\mathbb{Z}$, then $f^{\triangle}(t)$ reduces to the usual forward difference, that is $f^{\triangle}(t)=\triangle f(t)$. This theory not only brought equations leading to new applications. Also, this theory allows one to get some insight into and better understanding of the subtle differences between discrete and continuous systems [1, 2].

In this article we study some results related to integral inequalities by using Hölder's inequality on time scales.

Now, first we mention without proof several fundamental definitions and result from the calculus on time scales in an excellent introductory text by Bohner and Peterson [2].

\section{General Definitions}

Definition 1. A time scale $\mathbb{T}$ is a nonempty closed subset of $\mathbb{R}$. 
We assume throughout that $\mathbb{T}$ has the topology that is inherited from the standard topology on $\mathbb{R}$. It also assumed throughout that in $\mathbb{T}$ the interval $[a, b]$ means the set $\{t \in \mathbb{T}: s<t\}$ for the points $a<b$ in $\mathbb{T}$. Since a time scale may not be connected, we need the following concept of jump operators.

Definition 2. The mappings $\sigma, \rho: \mathbb{T} \rightarrow \mathbb{T}$ defined by $\sigma(t)=\inf \{s \in \mathbb{T}: s>t\}$ and $\rho(t)=\sup \{s \in \mathbb{T}: s<t\}$ are called the jump operators.

The jump operators $\sigma$ and $\rho$ allow the classification of points in $\mathbb{T}$ in the following way:

Definition 3. A nonmaximal element $t \in \mathbb{T}$ is said to be right-dense if $\sigma(t)=$ $t$, right-scattered if $\sigma(t)>t$, left-dense if $\rho(t)=t$, left-scattered if $\rho(t)<t$.

In the case $\mathbb{T}=\mathbb{R}$, we have $\sigma(t)=t$, and if $\mathbb{T}=h \mathbb{Z}, h>0$, then $\sigma(t)=t+h$.

Definition 4. The mapping $\mu: \mathbb{T} \rightarrow \mathbb{R}^{+}$defined by $\mu(t)=\sigma(t)-t$ is called the graininess function.

If $\mathbb{T}=\mathbb{R}$, then $\mu(t)=0$, and when $\mathbb{T}=\mathbb{Z}$, we have $\mu(t)=1$.

Definition 5. Let $f: \mathbb{T} \rightarrow \mathbb{R} . f$ is called differentiable at $t \in \mathbb{T}^{k}$, with (delta) derivative $f^{\triangle}(t) \in \mathbb{R}$ if given $\varepsilon>0$ there exists a neighborhood $U$ of $t$ such that, for all $s \in U$,

$\left\|f^{\sigma}(t)-f(s)-f^{\triangleright}(t)[\sigma(t)-s]\right\| \leq \varepsilon\|\sigma(t)-s\|$, where $f^{\sigma}=f \circ \sigma$.

If $\mathbb{T}=\mathbb{R}$, then $f^{\triangle}(t)=\frac{d f(t)}{d t}$, and if $\mathbb{T}=\mathbb{R}$, then $f^{\triangle}(t)=f(t+1)-f(t)$.

Some basic properties of delta derivatives are the following [2].

Theorem 1. Assume that $f: \mathbb{T} \rightarrow \mathbb{R}$ and let $t \in \mathbb{T}^{k}$.

(i) If $f$ is differentiable at $t$, then $f$ is continuous at $t$.

(ii) If $f$ is differentiable at $t$ and $t$ is right-scattered, then $f$ is differentiable at $t$ with

$$
f^{\triangle}(t)=\frac{f^{\sigma}(t)-f(t)}{\sigma(t)-t} .
$$

(iii) If $f$ is differentiable at $t$ and $t$ is right-dense, then

$$
f^{\triangle}(t)=\lim _{t \rightarrow s} \frac{f(t)-f(s)}{t-s} .
$$

(iv) If $f$ is differentiable at $t$, then

$$
f^{\sigma}(t)=f(t)+\mu(t) f^{\triangle}(t)
$$

Example 1. (i) If $f: \mathbb{T} \rightarrow \mathbb{R}$ is defined by $f(t)=\alpha$ for all $t \in \mathbb{T}$, where $\alpha \in \mathbb{R}$ is constant, then $f^{\triangle}(t) \equiv 0$.

(ii) If $f: \mathbb{T} \rightarrow \mathbb{R}$ is defined by $f(t)=t$ for all $t \in \mathbb{T}$, then $f^{\triangle}(t) \equiv 1$ 
Definition 6. The function $f: \mathbb{T} \rightarrow \mathbb{R}$ is said to be rd-continuous (denote $\left.f \in C_{r d}(\mathbb{T}, \mathbb{R})\right)$ if, at all $t \in \mathbb{T}$,

(i) $f$ is continuous at every right-dense point $t \in \mathbb{T}$,

(ii) $\lim _{s \rightarrow t^{-}} f(s)$ exists and is finite at every left-dense point $t \in \mathbb{T}$.

Definition 7. Let $f \in C_{r d}(\mathbb{T}, \mathbb{R})$. Then $g: \mathbb{T} \rightarrow \mathbb{R}$ is called the antiderivative of $f$ on $\mathbb{T}$ if it is differentiable on $\mathbb{T}$ and satisfies $g^{\triangle}(t)=f(t)$ for any $t \in \mathbb{T}^{k}$. In this case, we defined

$$
\int_{a}^{t} f(s) \triangle s=g(t)-g(a), \quad t \in \mathbb{T} .
$$

Theorem 2. If $f$ is $\triangle$-integrable on $[a, b]$, then so is $|f|$, and

$$
\left|\int_{a}^{b} f(t) \Delta t\right| \leq \int_{a}^{b}|f(t)| \triangle t
$$

Theorem 3. (Hölder Inequality). Let $a, b \in \mathbb{T}$. For $r d$-continuous functions $f, g:[a, b] \rightarrow \mathbb{R}$ we have

$$
\int_{a}^{b}|f(t) g(t)| \triangle t \leq\left(\int_{a}^{b}|f(t)|^{p} \triangle t\right)^{\frac{1}{p}}\left(\int_{a}^{b}|g(t)|^{q} \triangle t\right)^{\frac{1}{q}},
$$

where $p>1$ and $q=p /(p-1)$.

Theorem 4. (Cauch-Schwarz Inequality). Let $a, b \in \mathbb{T}$. For rd-continuous $f, g:[a, b] \rightarrow \mathbb{R}$ we have

$$
\int_{a}^{b}|f(t) g(t)| \Delta t \leq \sqrt{\left\{\int_{a}^{b}|f(t)|^{2} \Delta t\right\}\left\{\int_{a}^{b}|g(t)|^{2} \Delta t\right\}}
$$

Our main results are given in the following theorem.

\section{Main Result}

Proposition 5. Let $f, g$ be nonnegative functions with $0<m<f(x) / g(x) \leq$ $M$ on $[a, b]$. Then for $p>1$ and $q>1$ with $\frac{1}{p}+\frac{1}{q}=1$ we have

$$
\int_{a}^{b}[f(t)]^{1 / p}[g(t)]^{1 / q} \Delta t \leq M^{1 / p^{2}} m^{-1 / q^{2}}\left(\int_{a}^{b}[f(t)]^{1 / q}[g(t)]^{1 / p} \triangle t\right)
$$


and then,

$$
\int_{a}^{b}[f(t)]^{1 / p}[g(t)]^{1 / q} \Delta t \leq M^{1 / p^{2}} m^{-1 / q^{2}}\left(\int_{a}^{b} f(t) \Delta t\right)^{\frac{1}{q}}\left(\int_{a}^{b} g(t) \triangle t\right)^{\frac{1}{p}} .
$$

Proof. From Hölder's inequality, we obtain

$$
\int_{a}^{b}[f(t)]^{1 / p}[g(t)]^{1 / q} \Delta t \leq\left(\int_{a}^{b} f(t) \Delta t\right)^{\frac{1}{p}}\left(\int_{a}^{b} g(t) \Delta t\right)^{\frac{1}{q}}
$$

that is,

$$
\begin{aligned}
& \int_{a}^{b}[f(t)]^{1 / p}[g(t)]^{1 / q} \Delta t \\
\leq & \left(\int_{a}^{b}[f(t)]^{1 / p}[f(t)]^{1 / q} \triangle t\right)^{\frac{1}{p}}\left(\int_{a}^{b}[g(t)]^{1 / p}[g(t)]^{1 / q} \triangle t\right)^{\frac{1}{q}}
\end{aligned}
$$

Since $[f(t)]^{1 / p} \leq M^{\frac{1}{p}}[g(t)]^{1 / p}$ and $[g(t)]^{1 / q} \leq m^{-\frac{1}{q}}[f(t)]^{1 / q}$, from the above inequality it follows that

$$
\begin{aligned}
& \int_{a}^{b} f[(t)]^{1 / p}[g(t)]^{1 / q} \Delta t \\
\leq & M^{\frac{1}{p^{2}}} m^{-\frac{1}{q^{2}}}\left(\int_{a}^{b}[f(t)]^{1 / q}[g(t)]^{1 / p} \Delta t\right)^{\frac{1}{p}}\left(\int_{a}^{b}[f(t)]^{1 / q}[g(t)]^{1 / p} \Delta t\right)^{\frac{1}{q}}
\end{aligned}
$$

that is,

$$
\int_{a}^{b}[f(t)]^{1 / p}[g(t)]^{1 / q} \triangle t \leq M^{\frac{1}{p^{2}}} m^{-\frac{1}{q^{2}}} \int_{a}^{b}[f(t)]^{1 / q}[g(t)]^{1 / p} \triangle t
$$

Hence, the inequality (3.1) is proved.

The inequality (3.2) follows from substituting the following

$$
\int_{a}^{b}[f(t)]^{1 / q}[g(t)]^{1 / p} \Delta t \leq\left(\int_{a}^{b} f(t) \triangle t\right)^{\frac{1}{q}}\left(\int_{a}^{b} g(t) \triangle t\right)^{\frac{1}{p}}
$$

into (3.6), which can be obtained by Hölder's inequality. 
Proposition 6. For a given positive integer $p \geq 2$ if $0<m<f(x) \leq M$ on $\mathbb{T}$ with $M \leq m^{(p-1)^{2}} /(b-a)^{p}$, then

$$
\int_{a}^{b}[f(t)]^{1 / p} \Delta t \leq\left(\int_{a}^{b} f(t) \Delta t\right)^{1-\frac{1}{p}}
$$

Proof. Putting $g(t) \equiv 1$ into (3.2) yields

$$
\int_{a}^{b} f[(t)]^{1 / p} \Delta t \leq\left(\int_{a}^{b} f(t) \Delta t\right)^{1-\frac{1}{p}}
$$

where $K=M^{\frac{1}{p^{2}}}(b-a)^{\frac{1}{p}} / m^{\left(1-\frac{1}{p}\right)^{2}}$.

From $M \leq m^{(p-1)^{2}} /(b-a)^{p}$, we conclude that $K \leq 1$. Thus the inequality (3.8) is proved.

The special case $p=q=2$ yields the Cauchy-Schwarz inequality.

Proposition 7. Let $f, g$ be nonnegative functions with $0<m<f(x) / g(x) \leq$ $M$ on $\mathbb{T}$. We have

$$
\int_{a}^{b} f[(t)]^{1 / 2}[g(t)]^{1 / 2} \Delta t \leq M^{1 / 4} m^{-1 / 4}\left(\int_{a}^{b} f(t) \Delta t\right)^{\frac{1}{2}}\left(\int_{a}^{b} g(t) \Delta t\right)^{\frac{1}{2}} .
$$

Remark 1. Now we discuss a simple case of "equality" Proposition 2. If we make the substitution $f(t)=M=m$ and $b-a=1$ with $p=2$, then the equality in (3.8) holds.

Conclusion 1. If we take $\mathbb{T}=[a, b]$ in real interval in $\mathbb{R}$, it is clear that we can have the same Propositions. in ([4]).

\section{REFERENCES}

[1] R.P.Agarwal, M. Bohner, D. O'Regan, A. Peterson, Dynamic equations on time scale: A survey, J. Comput. Appl. Math. 141:1-26 (2002).

[2] M. Bohner, A. Peterson, Dynamic equations on time scale, An Introduction with Applications, Birkhauser, Boston, 2001.

[3] S. Hilger, analysis on measure chains- A unified approach to continuous and discrete calculus, result Math.18:18-56 (1990).

[4] L. Bougoffa, An integral inequality similar to Qi's inequality, J. İneq. in Pure and Appl. Math., 6 (2005), no.1, Article 27, (electronic).

Received: August 31, 2006 